\title{
FACTORIAL RATIOS THAT ARE INTEGERS
}

\author{
SAFWAN AKBIK \\ Hofstra University \\ Mathematics Department \\ Hempstead, NY 11554 \\ (Received March 15, 1993 and in revised form June 8, 1993)
}

\section{INTRODUCTION.}

The expressions

$$
\begin{gathered}
\frac{(2 n) !}{n !(n+1) !} \\
\frac{(2 r+1) !}{r !} \cdot \frac{(2 n) !}{n !(n+r+1) !} \\
s \cdot \frac{(2 n+s-1) !}{n !(n+s) !} \\
\frac{(s+2 r) !}{(s-1) ! r !} \cdot \frac{(2 n+s-1) !}{n !(n+s+r) !}
\end{gathered}
$$

are always integers. They are called the Catalan, generalized Catalan, ballot, and the super ballot numbers, respectively [1]. Here we consider two results concerning divisibility by expressions involving factorials, which generalize these and other similar assertions.

For given positive integers $a_{1}, a_{2}, \ldots, a_{t}$, let $\left\{a_{1}, a_{2}, \ldots, a_{t}\right\}$ denote the least common multiple of these integers. For integers $n$ and $k, n>k \geq 0$, set

$$
L(n, k)=\{n, n-1, \ldots, n-k\} .
$$

The novel aspect of our approach is the introduction of the function

$$
Q(J, B, C)=\prod_{i=0}^{J}(B-i, L(C, i)),
$$

for $B \geq C>J \geq 0$, where $(\alpha, \beta)$ denotes the greatest common divisor of the integers $\alpha$ and $\beta$.

Our results describe divisibility properties of this function "from above" and "from below". We have

THEOREM 1.1. let $m, k, J$, be positive integers such that $m \geq k>J \geq 0$, then the number $F(J, m, k)$ given by

$$
F(J, m, k)=\frac{Q(J, m, k)}{m(m-1) \ldots(m-J)} \cdot\left(\begin{array}{c}
m \\
k
\end{array}\right)
$$

is always an integer, where $\left(\begin{array}{c}m \\ k\end{array}\right)$ is the binomial coefficient.

THEOREM 1.2. For integers $s \geq 1, r \geq 0$, and $n \geq 1$, the integer

$$
P(r, s)=\frac{(2 r+s) !}{r !(s-1) !}
$$


is a multiple of

$$
Q(r, r+s+2 n, r+s+n) .
$$

Applying Theorem 1.1 with $J=0$, gives that for $m \geq k>0$,

$$
\frac{(m, k)}{m}\left(\begin{array}{c}
m \\
k
\end{array}\right)
$$

is an integer. (Note that (1.10) holds also for $k=0$.) Taking $m=2 n+s, k=n,(1.10)$ yields that

$$
\frac{(2 n+s, n)}{2 n+s}\left(\begin{array}{c}
2 n+s \\
n
\end{array}\right)=(2 n+s, n) \cdot \frac{(2 n+s-1) !}{n !(n+s) !}
$$

is an integer. Since $(2 n+s, n)=(s, n)$ divides $s$, we have that (1.3) is an integer. Then (1.1) is the special case $s=1$.

As for the expression (1.4), we apply Theorem 1.2, with $s \geq 1, r \geq 0$ and $n \geq 1$, obtaining that $P(r, s)$ is a multiple of $Q(r, r+s+2 n, r+s+n)$. But by Theorem 1.1,

$$
\begin{aligned}
& \frac{Q(r, r+s+2 n, r+s+n)}{(r+s+2 n)(r+s+2 n-1) \ldots(s+2 n)} \cdot\left(\begin{array}{c}
r+s+2 n \\
r+s+n
\end{array}\right) \\
& =Q(r, r+s+2 n, r+s+n) \cdot \frac{(s+2 n-1) !}{n !(r+s+n) !}
\end{aligned}
$$

is an integer. Thus (1.4) is an integer. Then (1.2) is the special case $s=1$.

2. PROOF OF THEOREM 1.1.

If not specified otherwise, all letters denote positive integers. Suppose that an integer $X$ is given as a product:

$$
X=\prod_{i=1}^{f} X_{\imath}
$$

For any positive integer $A$ we define

$$
N(A, X)=\text { the number of } X_{\imath} \text { divisible by } A .
$$

In all applications of this notation, the reference product $(2.1)$ will be uniquely given. For any prime $p$, let

$$
\text { Pow }(p, X)=\text { the largest } \alpha \text { such that } p^{\alpha} \text { divides } X
$$

It is easy to see that

$$
\operatorname{Pow}(p, X)=\sum_{\tau=1}^{\infty} N\left(p^{\tau}, X\right) .
$$

The following two lemmas are clear.

LEMMA 2.1. If $X$ is given by (2.1) and $Y=\prod_{j=1}^{h} Y_{j}$ is, such that, for all primes $p$ and $\tau>0$, we have

$$
N\left(p^{\tau}, Y\right) \geq N\left(p^{\tau}, X\right)
$$

then $X$ divides $Y$.

LEMMA 2.2. For $n \geq 1$, let $n !=\prod_{j=1}^{n} j$ be the reference product for $n$ !. Then

$$
N\left(p^{\tau}, n !\right)=\left[\frac{n}{p^{\tau}}\right]
$$


where $[a]$ is the number of positive integers $\leq a$.

From (1.7) we have

$$
F(J, m, k)=Q(J, m, k) \frac{(m-J-1) !}{k !(m-k) !} .
$$

Write $Q(J, m, k)$ in the form (2.1):

$$
Q(J, m, k)=\prod_{\imath=1}^{J}(m-i, L(k, i))=\prod_{\imath=0}^{J} Q_{\imath}(m, k) .
$$

By Lemmas 2.1 and 2.2, it is enough to show that

$$
N\left(p^{\tau}, Q\right)+\left[\frac{m-J-1}{p^{\tau}}\right] \geq\left[\frac{m-k}{p^{\tau}}\right]+\left[\frac{k}{p^{\tau}}\right] .
$$

Set

$$
\Delta\left(p^{\tau}, F\right)=N\left(p^{\tau}, Q\right)+\left[\frac{m-J-1}{p^{\tau}}\right]-\left[\frac{m-k}{p^{\tau}}\right]-\left[\frac{k}{p^{\tau}}\right],
$$

so that (2.9) is equivalent to

$$
\Delta\left(p^{\tau}, F\right) \geq 0
$$

Let

$$
\frac{m-k}{p^{\tau}}=\left[\frac{m-k}{p^{\tau}}\right]+\frac{d_{\tau}}{p^{\tau}}, \quad \frac{k}{p^{\tau}}=\left[\frac{k}{p^{\tau}}\right]+\frac{e_{\tau}}{p^{\tau}},
$$

where,

$$
0 \leq d_{\tau} \leq p^{\tau}-1, \quad 0 \leq e_{\tau} \leq p^{r}-1
$$

Then

$$
\frac{m-J-1}{p^{\tau}}=\left[\frac{m-k}{p^{\tau}}\right]+\left[\frac{k}{p^{\tau}}\right]+\frac{d_{\tau}+e_{\tau}-J-1}{p^{\tau}},
$$

implying

$$
\left[\frac{m-J-1}{p^{\tau}}\right]=\left[\frac{m-k}{p^{\tau}}\right]+\left[\frac{k}{p^{\tau}}\right]+\left[\frac{d_{\tau}+e_{\tau}-J-1}{p^{\tau}}\right]
$$

From (2.10) and (2.15) we have:

$$
\Delta\left(p^{\tau}, F\right)=N\left(p^{\tau}, Q\right)+\left[\frac{d_{\tau}+e_{\tau}-J-1}{p^{\tau}}\right] .
$$

If $d_{\tau}+e_{\tau}-J-1 \geq 0$, then $\Delta\left(p^{\tau}, F\right) \geq 0$. Suppose that $d_{\tau}+e_{\tau}-J-1<0$, then $d_{\tau}+e_{\tau} \leq J$. If

$$
L=d_{\tau}+e_{\tau},
$$

$0 \leq L \leq J$. By (2.12) we have that $p^{\tau}$ divides both $m-k-d_{\tau}$ and $k-e_{\tau}$, and hence it divides $m-\left(d_{\tau}+e_{\tau}\right)=m-L$. Then $p^{\tau}$ divides $\left(m-L, k-e_{\tau}\right)$. For $t \geq 0$ we have

$$
p^{\tau} \mid\left(m-L-t p^{\tau}, k-e_{r}\right) .
$$

For each $t$ such that $L+t p^{\tau} \leq J, p^{\tau}$ divides:

$$
\left(m-L-t p^{\tau},\left\{k, k-1, \ldots, k-e_{\tau}, \ldots, k-L-t p^{\tau}\right\}\right)=Q_{L+t p^{\tau}}(m, k) .
$$

Thus each $0 \leq t \leq\left[\frac{J-L}{p^{\tau}}\right]$ maps onto $Q_{L+t p^{r}}(m, k)$ that is divisible by $p^{\tau}$. Since this map is 1-1 into the factors $Q_{\imath}(m, k)$ in (2.8) that are divisible by $p^{\tau}$, it follows that

$$
N\left(p^{\tau}, Q\right) \geq 1+\left[\frac{J-L}{p^{\tau}}\right] .
$$


From (2.16), (2.17), and (2.19) we have

$$
\Delta\left(p^{\tau}, F\right) \geq 1+\left[\frac{J-L}{p^{\tau}}\right]+\left[\frac{L-J-1}{p^{\tau}}\right]
$$

It is casy to see that

$$
\left[\frac{L-J-1}{p^{\tau}}\right]=-\left[\frac{J-L}{P^{\tau}}\right]-1 .
$$

Since (2.20) and (2.21) imply (2.11), Theorem 1.1 is proved.

3. PROOF OF THEOREM 1.2 .

LEMMA 3.1. Let

$$
U=\prod_{l=1}^{a} U_{\imath}, \quad V=\prod_{\jmath=1}^{b} V_{\jmath}, \quad W=\prod_{l=1}^{c} W_{l}, \quad Z=\prod_{k=1}^{d} Z_{k} .
$$

For all primes $p$ and integers $\tau>0$, we assume that

$$
N\left(p^{\tau}, W\right) \leq \min \left(N\left(p^{\tau}, U\right), N\left(p^{\tau}, V\right)\right),
$$

and

$$
N\left(p^{\tau}, Z\right) \leq \max \left(N\left(p^{\tau}, U\right), N\left(p^{\tau}, V\right)\right) .
$$

Then $\frac{U V}{W}$ is an integer divisible by $Z$.

PROOF. We have for any prime $p$,

$$
\operatorname{Pow}\left(p, \frac{U V}{W}\right)=\sum_{\tau=1}^{\infty}\left(N\left(p^{\tau}, U\right)+N\left(p^{\tau}, V\right)-N\left(p^{\tau}, W\right)\right)
$$

Let

$$
\lambda\left(p^{\tau}\right)=N\left(p^{\tau}, U\right)+N\left(p^{\tau}, V\right)-N\left(p^{\tau}, W\right) .
$$

Via (3.2) and (3.3) we have:

$$
\begin{aligned}
\lambda\left(p^{\tau}\right)= & \max \left(N\left(p^{\tau}, U\right), N\left(p^{\tau}, V\right)\right)+\min \left(N\left(p^{\tau}, U\right), N\left(p^{\tau}, V\right)\right)-N\left(p^{\tau}, W\right) \\
& \geq N\left(p^{\tau}, Z\right)+N\left(p^{\tau}, W\right)-N\left(p^{\tau}, W\right)=N\left(p^{\tau}, Z\right) .
\end{aligned}
$$

This and (3.4) yield

$$
\operatorname{Pow}\left(p, \frac{U V}{W}\right) \geq \sum_{\tau=1}^{\infty} N\left(p^{\tau}, Z\right)=\operatorname{Pow}(p, Z),
$$

and the lemma follows.

Write (1.9) in the form:

$$
Q(r, r+s+2 n, r+s+n)=\prod_{k=0}^{r} Q_{k}
$$

where

$$
Q_{k}=(r+s+2 n-k, L(r+s+n, k)) .
$$

We also rewrite (1.8) in the form:

$$
P(r, s)=\prod_{i=0}^{r-1}(2 r+s-i) \prod_{j=0}^{r}(r+s-j) / r !
$$


We will obtain Theorem 1.2 by applying Lemma 3.1 with

$$
\begin{aligned}
& U=\prod_{i=0}^{r-1} U_{i}=\prod_{i=0}^{r-1}(2 r+s-i), \\
& V=\prod_{j=0}^{r} V_{J}=\prod_{j=0}^{r}(r+s-j), \\
& W=\prod_{l=0}^{r-1} W_{l}=\prod_{l=0}^{r-1}(l+1)=r ! \\
& Z=\prod_{k=0}^{r} Z_{k}=\prod_{k=0}^{r} Q_{k} .
\end{aligned}
$$

Thus $Z=Q(r, r+s+2 n, r+s+n)=Q$, and

$$
P(r, s)=\frac{U V}{W}=\left(\begin{array}{c}
2 r+s \\
r
\end{array}\right) \frac{(r+s) !}{(s-1) !}
$$

is an integer. As for (3.2) we have:

$$
\begin{aligned}
& N\left(p^{\tau}, W\right)=N\left(p^{\tau}, r !\right)=\left[\frac{r}{p^{\tau}}\right], \\
& N\left(p^{\tau}, U\right)=\left[\frac{2 r+s}{p^{\tau}}\right]-\left[\frac{r+s}{p^{\tau}}\right], \\
& N\left(p^{\tau}, V\right)=\left[\frac{r+s}{p^{\tau}}\right]-\left[\frac{s-1}{p^{\tau}}\right],
\end{aligned}
$$

from which the inequalities $N\left(p^{\tau}, W\right) \leq N\left(p^{\tau}, U\right), N\left(p^{\tau, W}\right) \leq N\left(p^{\tau}, V\right)$ are obvious. Thus the proof reduces to establishing (3.3). Consider those $Q_{k}, 0 \leq k \leq r$, such that $p^{r}$ divides $Q_{k}$. Since this requires that $p^{\tau}$ divides

$$
L(r+s+n, k)=\{r+s+n, r+s+n-1, \ldots, r+s+n-k\},
$$

the smallest $k$ for which this occurs is $\mu^{*}$, where

$$
r+s+n-\mu^{*} \equiv 0 \quad\left(p^{\tau}\right), \quad 0 \leq \mu^{*}<p^{\tau}, \quad \mu^{*} \leq r .
$$

(It is the last inequality that constrains, in part, the existence of such a $Q_{k}$.) Also, there would be a smallest $k^{*}, \mu^{*} \leq k^{*} \leq r$, such that

$$
r+s+2 n-k^{*} \equiv 0 \quad\left(\bmod p^{\tau}\right) .
$$

From (3.8) and (3.9) we have

$$
n \equiv k^{*}-\mu^{*} \quad\left(\bmod p^{\tau}\right)
$$

Thus (3.9) is equivalent to

$$
r+s+2\left(k^{*}-\mu^{*}\right)-k^{*} \equiv 0 \quad\left(\bmod p^{r}\right), \quad \mu^{*} \leq k^{*} \leq r .
$$

If (3.8) and (3.11) are not satisfied then $N\left(p^{\tau}, Z\right)=N\left(p^{\tau}, Q\right)=0$, and (3.3) certainly holds. Thus we may assume that $N\left(p^{\tau}, Q\right)>0$. The integers $k$ such that $p^{\tau}$ divides $Q_{k}$ are precisely those such that

$$
k^{*} \leq k \leq r, \quad k \equiv k^{*} \quad\left(\bmod p^{r}\right)
$$


which given

$$
N\left(p^{\tau} \cdot Q\right)=1+\left[\frac{1-k^{*}}{p^{\tau}}\right]
$$

Comsider two cases:

Casc I. $\mu^{*} \leq k^{*} \leq 2 \mu^{*}$. Here, for all $k$ satisfying (3.12), wo have

$$
r+s+2\left(k^{*}-\mu^{*}\right)-k \leq r+s+2\left(k^{*}-\mu^{*}\right)-k^{*} \leq r+s,
$$

and

$$
r+s+2\left(k^{*}-\mu^{*}\right)-k \geq r+s+2\left(k^{*}-\mu^{*}\right)-r \geq s .
$$

Note that this implies

$$
r \geq k-2\left(k^{*}-\mu^{*}\right) \geq 0 .
$$

Thus, in this case. a factor $Q_{k}$ which is divisible by $p^{\tau}$ maps onto $V_{k-2\left(k^{*}-\mu^{*}\right)}$, which is divisible by $p^{r}$. Since this map is 1-1 into the set of $V$, that are divisible by $p^{r}$, we have

$$
N\left(p^{\tau}, V\right) \leq N\left(p^{\tau}, Q\right)
$$

Case II. $k^{*}>2 \mu^{*}$. Let

$$
q^{*}=r+s+k^{*}-2 \mu^{*} .
$$

By $(3.11)$, we have $q^{*} \equiv 0\left(\bmod p^{r}\right)$. Also

$$
q^{*} \geq r+s+1
$$

and

$$
q^{*} \leq r+s+k^{*} \leq 2 r+s .
$$

Thus $q^{*}$ is one of the $U_{t}$ and is divisible by $p^{\tau}$. Hence the integers of the form $q^{*}+t p^{r}$ such that

$$
q^{*}+t p^{\tau} \leq 2 r+s, \quad t \geq 0,
$$

are also among the $U_{\imath}$ 's, which are divisible by $p^{\tau}$. This yields

$$
N\left(p^{\tau}, U\right) \geq 1+\left[\frac{2 r+s-q^{*}}{p^{\tau}}\right]
$$

or inserting (3.15),

$$
N\left(p^{\tau}, U\right) \geq 1+\left[\frac{r-k^{*}+2 \mu^{*}}{p^{\tau}}\right] .
$$

(Actually equality can be proved in (3.18), but this is not needed). Via (3.13) and (3.18), the inequality

$$
N\left(p^{\tau}, Q\right) \leq N\left(p^{\tau}, U\right)
$$

would be a consequence of

$$
\left[\frac{r-k^{*}}{p^{\tau}}\right] \leq\left[\frac{r-k^{*}+2 \mu^{*}}{p^{\tau}}\right]
$$

But the last inequality is obvious since $\mu^{*} \geq 0$, and the theorem follows.

\section{REFERENCES}

1. Ira M. Gessel, Super Ballot Numbers, Brandeis University, Preprint.

2. Richard K. Guy, Unsolved Problems in Number Theory, Springer Verlag, NY, 1981, pp. 49. 


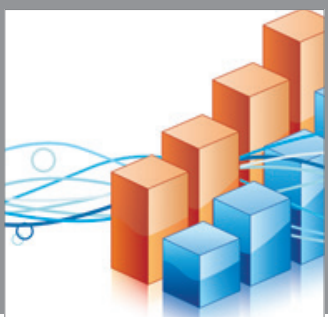

Advances in

Operations Research

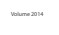

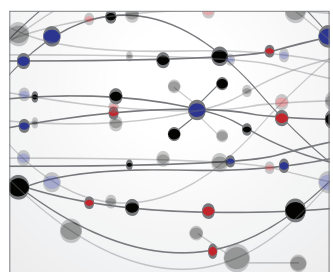

\section{The Scientific} World Journal
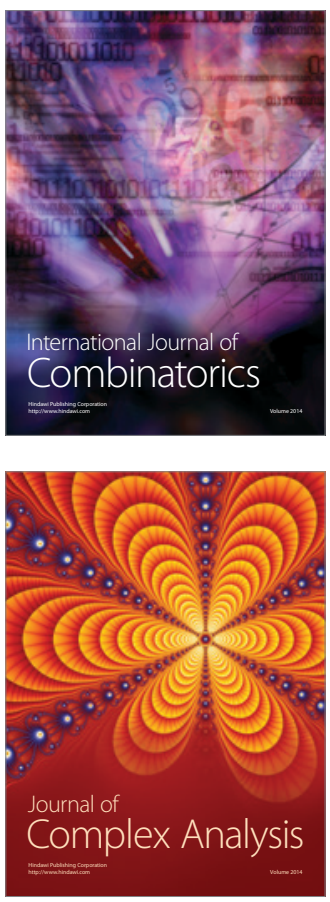

International Journal of

Mathematics and

Mathematical

Sciences
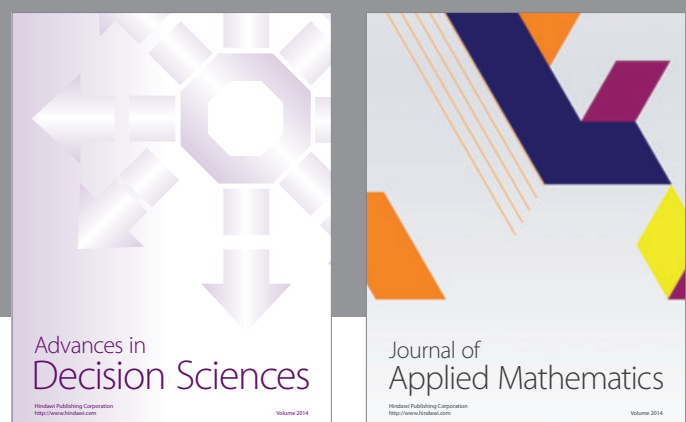

Journal of

Applied Mathematics
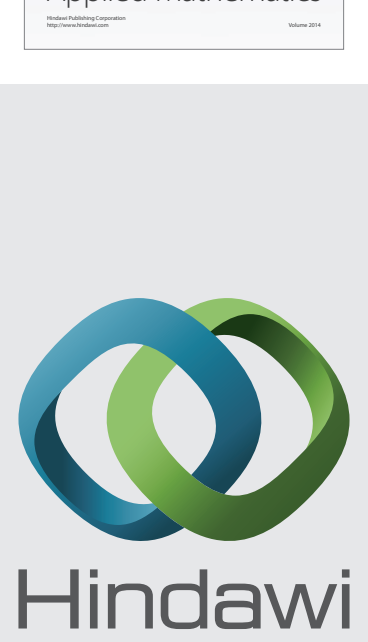

Submit your manuscripts at http://www.hindawi.com
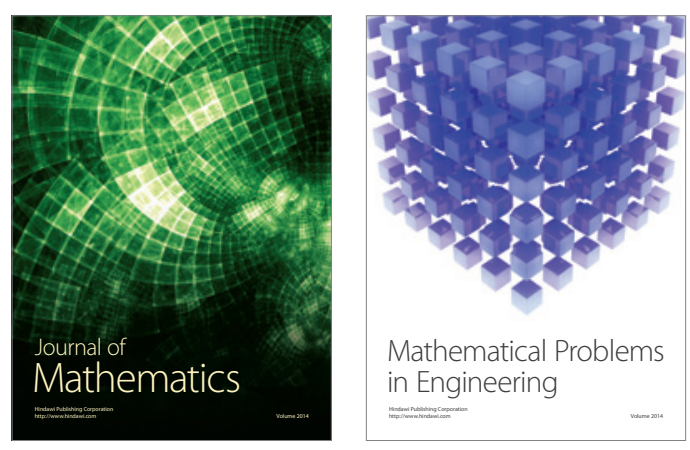

Mathematical Problems in Engineering
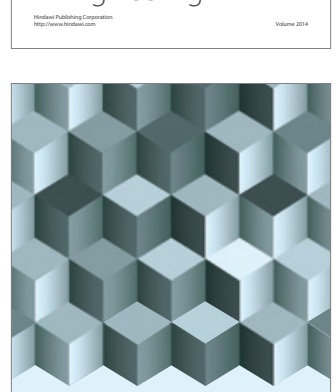

Journal of

Function Spaces
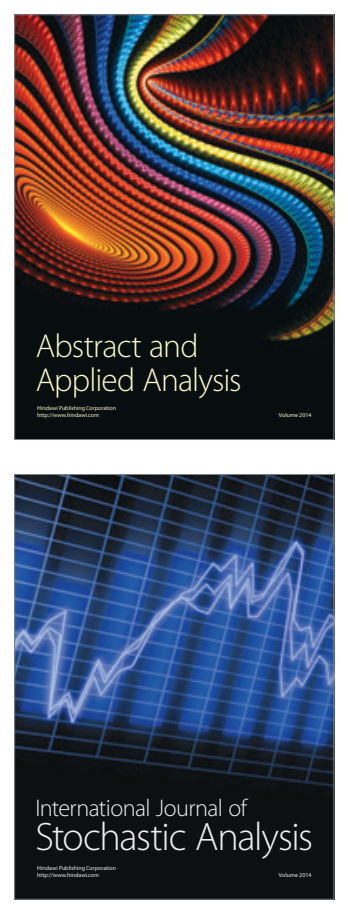

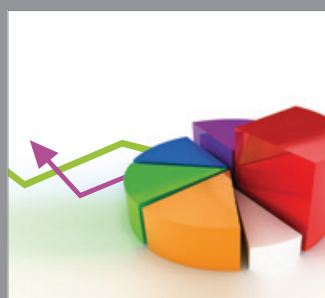

ournal of

Probability and Statistics

Promensencen
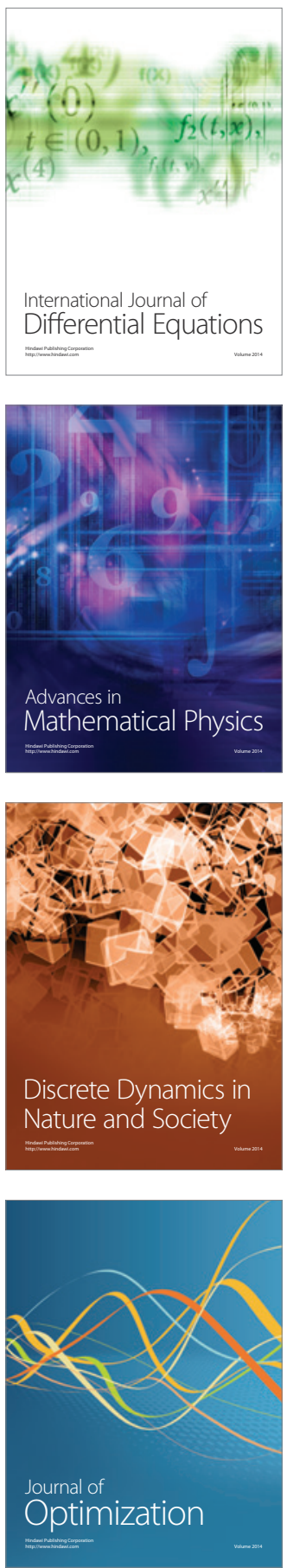